\title{
Dust formation in evolved stars and supernovae: new advances and unsolved problems
}

\author{
Isabelle Cherchneff \\ Dept. of Physics, Basel University, \\ CH-4056 Basel, Swizerland \\ email: isabelle.cherchneff@unibas.ch
}

\begin{abstract}
This paper briefly reviews some advances and unsolved problems related to the formation of cosmic dust in the evolved environments of AGB stars and supernovae.
\end{abstract}

Keywords. stars: AGB and post-AGB, supernovae: general, circumstellar matter, molecular processes

\section{Introduction}

Sources of cosmic dust in the universe include evolved low- and high- mass stars and core-collapse supernovae (SNe). These environments, specifically the winds of stars and the material ejected by $\mathrm{SNe}$, are all characterised by shock activity and high gas densities and temperatures. These conditions are necessary to the efficient formation of molecular clusters, and their growth through coalescence, coagulation and accretion to form dust grains. The Spitzer and Herschel space missions provided new insights on the type and quantity of dust produced by evolved stars and SNe. These advances are now tested with ALMA. New theoretical models also provide a better understanding of the processes that control dust formation. However, there are still many unsolved issues that relate to the chemical nature of the condensates and the mechanisms that trigger their formation. The majority of existing models of dust formation in stars rely on thermodynamic equilibrium (TE) predictions (e.g., Tielens 1998, Lodders \& Fegley 1999) or chemical equilibrium assumptions underpinning the formalism and use of the "Classical Nucleation Theory" (e.g., Gail \& Sedlmayr 1999, Ferrarotti \& Gail 2006, Woitke 2006, Freytag \& Höfner 2008, Bladh \& Höfner 2012, Nanni et al. 2013, Dell'Agli et al. 2014). Because shocks shape the dust formation regions in stellar sources, non-equilibrium conditions prevail and command the processes controlling dust synthesis. Specifically, the chemistry responsible for the growth of dust clusters of any chemical type is not at equilibrium and probably involves pathways similar to those encountered in various dust-producing laboratory experiments (e.g., flame, pyrolysis and shock tube). The characterisation of the most stable dust clusters requires further investigation, and the chemical routes operating at high gas temperature and leading to cluster formation need to be identified.

\section{Asymptotic Giant Branch stars}

Recent mid-infrared (IR) interferometric data of oxygen-rich (O-rich) AGB stars indicate the presence of dust, possibly alumina $\left(\mathrm{Al}_{2} \mathrm{O}_{3}\right)$, close to the stellar photosphere between $1 \mathrm{R}_{\star}$ and $2 \mathrm{R}_{\star}$ (e.g., Norris et al. 2012, Zhao-Geisler et al. 2012, Karovicova et al. 2013). In the most evolved objects, the addition of a shell of silicate dust located 
at $\sim 3-5 \mathrm{R}_{\star}$ is necessary to reproduce the data (Karovicova et al. 2013). These results clearly indicate a trend in dust formation on the AGB, whereby the less evolved object, which are hotter, form highly refractory condensates like alumina close to the stellar surface, while the more evolved, cooler stars of Mira-type prevalently form silicates at larger radii, along with forming alumina in the deep layers of their wind. Such trends in dust formation sequences were already derived from the analysis of IRAS spectra of O-rich AGBs by Stencel et al. (1990). A recent model based on chemical kinetics confirms these trends for the Mira star IK Tau (Gobrecht et al. 2015). The gas phase and the nucleation of dust clusters in the periodically shocked layers above the photosphere are modelled with non-TE chemistry and coupled to the condensation of clusters through coalescence and coagulation. Nucleation chemistry for both alumina and silicate is evolved from $1 \mathrm{R}_{\star}$ to $10 \mathrm{R}_{\star}$ in the shocked inner wind. Alumina forms close to the star $\left(r \leqslant 2 \mathrm{R}_{\star}\right)$, while silicates form at $r \geqslant 3.5 \mathrm{R}_{\star}$. Both dust components form from the gas phase and do not require heterogeneous condensation on pre-existing nuclei (e.g., $\mathrm{TiO}_{2}$ ) to proceed, albeit this process may occur. One pending problem relates to the wind acceleration efficiency in O-rich AGBs, as alumina and silicates are transparent material. The fate of iron and its partial inclusion into Fe-rich silicates is thus a topical issue that is worth investigating.

\section{Supernovae}

Dust formation in the ejecta of Type II-P SNe was first observed in the IR at day $\sim 500$ post-explosion in SN1987A (e.g., Lucy et al. 1989). The dust IR thermal emission has since been observed in other SNe (e.g., Kotak et al. 2009) and points to modest masses of condensates in the ejecta $\left(\sim 10^{-5}-10^{-3} \mathrm{M}_{\odot}\right)$. In contrast, the submillimetre fluxes measured with Herschel and ALMA in the young SN1987A remnant and in Cas A indicate large quantities of dust $\left(\sim 0.1-0.5 \mathrm{M}_{\odot}\right)$ in the remnant phase (e.g., Barlow et al. 2010, Matsuura et al. 2011, Indebetouw et al. 2014). Several scenarios are proposed to explain the dust mass discrepancy in the two SN phases. Wesson et al. (2015) propose a continuous growth of dust grains in SN1987A over a 28 year period, albeit the mechanisms responsible for grain growth at late times are not identified. Cold accretion of species onto dust grains and coagulation and coalescence processes are rather inefficient on short timescales and at low gas densities. Sarangi \& Cherchneff (2015) model dust formation in various Type II-P SNe and show that several dust components, including silicates, alumina, carbon and iron, gradually form in different regions of the ejecta over $\sim 3-5$ years after explosion. Finally, Dwek \& Arendt (2015) propose a large quantity of enstatite dust $\left(\sim 0.4 \mathrm{M}_{\odot}\right)$ forms at early time and remains hidden in clumps, along with a small carbon dust component. The choice of enstatite is driven by reproducing dust emission fluxes, but is not supported by models, which find that forsterite is the prevalent silicate in the ejecta (Sarangi \& Cherchneff 2015). More observations of SNe in their transition phase are required to settle this debated issue.

Molecules, including CO, SiS, $\mathrm{SiO}, \mathrm{SO}$ and $\mathrm{CS}$, are predicted to form in large quantities in SN ejecta (Lepp et al. 1990, Cherchneff \& Dwek 2009, Sarangi \& Cherchneff 2013). The latter study shows the species trace different ejecta zones, control the quantity of elements available for dust formation and may even partake in the synthesis of dust. Hence, the large mass of CO detected with ALMA in SN1987A does not come as a surprise (Kamenetzky et al. 2013). The detection of molecules other than CO with ALMA is still awaiting confirmation. More generally, the chemistry of SN ejecta provides direct information on the mixing in the ejecta and on the dust chemical nature. Therefore, the molecular content of SN explosions requires further astronomical investigation. 


\section{References}

Barlow, M. J., Krause, O., Swinyard, B. M. et al. 2010, A\&A, 518, L138

Bladh, S. \& Höfner, S. 2012, A\&\&A, 546, A76

Cherchneff, I. \& Dwek, E. 2009, ApJ, 703, 642

Dell'Agli, F., Garcia-Hernández, D. A., Rossi. C. et al. 2014, M.N.R.A.S., 441, 1115

Ferrarotti, A. S. \& Gail, H.-P 2006, A\&AA, 447, 553

Freytag, B. \& Höfner, 2008, $A \mho A, 483,571$

Gail, H-P. \& Sedlmayr, E. 1999, A\&\&A, 347, 594

Gobrecht, D., Cherchneff, I. Sarangi, A. et al. 2015, arXiv:1509.07613

Indebetouw, R., Matsuura, M., Dwek, E. et al. 2014, ApJ (Letters), 782, L2.

Kamenetzky, J., McCray, R., Indebetouw, R. et al. 2013, ApJ (Letters), 773, L34

Karovicova, I., Wittkowski, M., Ohnaka, K. et al. 2013, A\&\&A, 560, A75

Kotak, R., Meikle, W. P. S., Farrah, D. et al. 2009, ApJ, 704, 306

Lepp, S., Dalgarno, A., \& McCray, R. 1990, ApJ, 358, 262

Lodders, K. \& Fegley, B. 1999, in T. Le Bertre, A. Lèbre, \& C. Waelkens (eds.), IAU Symposium 191 'Asymptotic Giant Branch Stars,', p 279

Lucy, L. B., Danziger, I. J., Gouiffes, C., \& Bouchet, P. 1989, in G. Tenorio-Tagle, M. Moles \& J. Melnick (eds.), IAU Symposium 120 'Structure and Dynamics of the Interstellar Medium', p. 164

Matsuura, M., Dwek, E., Meixner, M. et al. 2011, Science, 333, 1258

Meyer, B. S., Clayton, D. D., \& The, L.-S. 2000, ApJ (Letters), 540, L49

Nanni, A., Bressan, A., Marigo, P., \& Girardi, L. 2013, M.N.R.A.S., 434, 2390

Norris, B. R. N., Tuthill, P. G., Ireland, M. J. et al. 2012, Nature, 484, 220

Sarangi, A. \& Cherchneff, I. 2013, ApJ, 776, 107

Sarangi, A. \& Cherchneff, I. 2015, A\&A, 575, A95

Stencel, R. E., Nuth, J. A., Little-Marenin, I. R., \& Little, S. J. 1990, ApJ (Letters), 350, L45

Tielens 1998, A\&SS, 255, 415

Woitke, P. 2006, A\&A, 460, L9

Zhao-Geisler, R. Quirrenbach, A., Köler, R., \& Lopez, B. 2012, A\&A, 545, A56 